\title{
An Observational Study of QTc Prolongation in Critically Ill Patients: Identification of Incidence and Predictors
}

\author{
Behrooz Farzanegan ${ }^{1}$, Zeinab Hosseinpoor ${ }^{2}$, Shadi Baniasadi ${ }^{3}$, Seyyed R Seyyedi $^{4}$, Mehdi Rajabi $^{5}$
}

\begin{abstract}
Aims: Despite the importance of abnormal QTc interval values in intensive care unit (ICU) patients, there is a paucity of information on this topic. The current study was designed to identify the incidence and predictors of QTc prolongation in medical (M), surgical (S), and emergency (E) ICUs. Materials and methods: A prospective observational study was conducted for 6 months. Patients more than 18 years old who admitted to MICU, $\mathrm{SICU}$, and EICU were included in the study. Electrocardiogram (ECG) was taken on day 1, 3, and 5 of ICU admission. The QTc intervals $>460 \mathrm{~ms}$ in male and $>470 \mathrm{~ms}$ in female and increased $>60 \mathrm{~ms}$ above baseline were considered QTc prolongation. Comparative analysis was done between two groups of patients (normal vs prolonged QTc). Logistic regression models were carried out to determine the predictors of QTc prolongation. Results: Incidence of QTc prolongation was 6.5, 9.8, and $15.7 \%$ on day 1, 3, and 5 of ICU admission, respectively. On day 1, the history of alcohol addiction and the reason of ICU admission were associated with a prolonged QTC. A significant association was demonstrated between administration of azithromycin and QTc prolongation on day 3. High serum creatinine and hospitalization in EICU were predictors of QTC prolongation on day 5 of ICU admission.
\end{abstract}

Conclusion: The QTC prolongation is relatively common among patients admitted to ICUs and its incidence increases with increasing length of hospital stay. Predictors of QTc prolongation may be affected by the duration of ICU admission. Physicians should consider these predictors particularly before prescribing QTc-prolonging drugs.

Keywords: Critically ill patient, Intensive care unit, QTc prolongation.

Indian Journal of Critical Care Medicine (2020): 10.5005/jp-journals-10071-23411

\section{INTRODUCTION}

About 17.9 million people die yearly from cardiovascular diseases across the world. ${ }^{1}$ Some cardiovascular deaths are the result of arrhythmias. The majority of cardiac arrests are attributed to malignant cardiac arrhythmias and heart rhythm problems that are common reason for admission to intensive care units (ICUs). ${ }^{2}$ The QTc prolongation is a consequence of delayed ventricular repolarization and may lead to a potentially life-threatening arrhythmia, torsade de pointes (Tdp). ${ }^{3}$

Drug therapy is the most common cause of QTc prolongation. ${ }^{4}$ Several antiarrhythmic agents, fluoroquinolones, phenothiazine antipsychotics, butyrophenones, macrolides, antihistamines, and tricyclic antidepressants are known to prolong QTc interval. ${ }^{5}$ All drugs prolonging the QTc interval act directly on ion channels of myocytes or indirectly reduce the elimination of QTc-prolonging drugs by affecting hepatic metabolism. ${ }^{6}$ Despite the risk of QTC prolongation by these medications, they are commonly prescribed by physicians without increased vigilance. ${ }^{7}$ In the last decade, druginduced QTc prolongation is one of the most common causes of relabeling or removing medications from the markets. ${ }^{8}$

Risk factors for QTc prolongation and Tdp are female sex, old age, cardiac disease, electrolyte abnormalities, diabetes mellitus type II, thyroid disease, and bradycardia., ${ }^{9} 10$ Females are at higher risk of developing QTC prolongation than males; this is probably because estrogen lengthens the QTc interval and testosterone shortens it. ${ }^{11,12}$ Patients with older age are at higher risk of QTc prolongation, which may be relevant to decreasing sex hormones. ${ }^{13,14}$ Hyperglycemia has an effect on cardiac repolarization and may contribute to a longer QTc interval or cardiac arrhythmia. ${ }^{15,16}$ The QTc intervals normally shorten with tachycardia and lengthen with bradycardia. Therefore, bradycardia is a risk factor for developing QTc prolongation. ${ }^{5}$
${ }^{1,3}$ Tracheal Diseases Research Center, National Research Institute of
Tuberculosis and Lung Diseases, Shahid Beheshti University of Medical
Sciences, Tehran, Iran
${ }^{2,5}$ Department of Clinical Pharmacy, Faculty of Pharmacy, Tehran
Medical Sciences, Islamic Azad University, Tehran, Iran
${ }^{4}$ Chronic Respiratory Diseases Research Center, National Research
Institute of Tuberculosis and Lung Diseases, Shahid Beheshti University
of Medical Sciences, Tehran, Iran

Corresponding Author: Mehdi Rajabi, Department of Clinical Pharmacy, Faculty of Pharmacy, Tehran Medical Sciences, Islamic Azad University, Tehran, Iran, Phone: +98-21-22640056, e-mail: mehdirj@ aol.co.uk

How to cite this article: Farzanegan B, Hosseinpoor Z, Baniasadi S, Seyyedi SR, Rajabi M. An Observational Study of QTc Prolongation in Critically III Patients: Identification of Incidence and Predictors. Indian J Crit Care Med 2020;24(4):270-275.

Source of support: National Research Institute of Tuberculosis and Lung Diseases, Shahid Beheshti University of Medical Sciences, Tehran, Iran

Conflict of interest: None

Critically ill patients are more prone to develop QTc prolongation during ICU stay because of exposure to various risk factors and administration of QT-prolonging drugs. ${ }^{17}$ Awareness of risk factors and prevention of QTC prolongation are very important in ICU patients to avoid hospital deaths. ${ }^{18}$ In other words, identifying patients with high risk of QTc prolongation and avoidance of administration of QT-prolonging drugs or occurrence of drugdrug interactions are simple and cost-effective way of decreasing hospital mortality. ${ }^{19}$ 
Although QTc prolongation in ICU patients is an important issue of the study, a paucity of information is available concerning the incidence and predictors of QTc prolongation in different days of ICU admission. ${ }^{7}$ Moreover, only few studies are available that follow patients until the point of discharged from ICU, and little research has been conducted in emergency ICU. Therefore, current study was designed to identify the incidence and predictors of QTC interval prolongation during hospitalization in MICU, SICU, and EICU. Patients with normal and prolonged QTc intervals were also compared in terms of clinical characteristics and risk factors of QTC prolongation. All patients with QTc prolongation were followed during their hospital stay.

\section{Materials and Methods}

\section{Study Design}

This prospective observational study was conducted for 6 months in National Research Institute of Tuberculosis and Lung Diseases (NRITLD), an affiliated university center for education, research, and therapy. The study protocol was approved by the ethical review board of NRITLD. Patients above the age of 18 who were admitted to MICU, SICU, and EICU were included in the study. Patients with pacemaker or paced rhythms, history of long QT syndromes, discharge from ICU in less than 24 hours, or no follow-up ECG were excluded from the study. All patients were evaluated with a 12-lead ECG on day 1, 3, and 5 of admission in ICU. If the ICU admission prolonged more than 5 days, patients were monitored by ECG every 5 days thereafter. All patients with QTc prolongation were followed regarding ECG abnormalities during hospital stay.

\section{Measurement and Calculation of QTc Interval}

The QTc intervals were measured from beginning of $Q$ to the end of T wave. ${ }^{20}$ The $U$ wave was not included in the measurement of QTC interval in our study. ${ }^{7}$ Heart rate affects the QT interval, and various correction formulas are used to normalize it. ${ }^{21}$ Bazett's formula is the most common ones used by physicians for correction of QT interval. However, overestimation is under debate as the heart rate deviates from 60 beats/minute. ${ }^{22}$ It has been shown that Hodges formula is the best choice for correction of QT interval. ${ }^{23}$ Therefore, the formula used for calculation of corrected QTc interval was Hodges $[\mathrm{QTC}=\mathrm{QT}+1.75(\mathrm{HR}-60)]$.

The QTc intervals $>460 \mathrm{~ms}$ in male and $>470 \mathrm{~ms}$ in female and increased $>60$ ms above baseline (day 1 of ICU admission) were considered QTc prolongation. ${ }^{24}$

\section{Data Collection}

The following information was collected for the patients: baseline demographics, medications, age, laboratory findings, acute physiologic and chronic health evaluation (APACHE II), and the duration of hospitalization. In addition, risk factors for QTc prolongation including hypokalemia, hypocalcemia, hypomagnesemia, bradycardia, congestive heart failure, female gender, kidney or liver disease, thyroid disorders, diabetes mellitus, arrhythmia, cerebral aneurysm, left ventricular ejection fraction of less than $45 \%$ (LVEF $<45 \%$ ), coronary artery disease, pulmonary embolism, deep vein thrombosis (DVT), valvular heart disease, infections, chronic obstructive pulmonary disease, history of stroke or myocardial infarction, surgery, percutaneous coronary intervention ( $\mathrm{PCl}$ ), hypertension, smoking, and alcohol consumption were gathered for each patient. ${ }^{9,10}$ The International Classification of Diseases, 10th edition (ICD-10) was used to classify the reason of admission to the ICUs. ${ }^{25}$

\section{Medications with QTc Prolongation Effects}

All medications administered to the patients were categorized in terms of QTc prolongation potency based on the information from CredibleMeds website. This website categorizes drugs into a broad range based on predisposing risk of QTc prolongation or Tdp. Drugs in known risk category prolong the QTC interval and are evidently associated with Tdp, even they are taken as recommended. Drugs in possible risk category can cause QT prolongation but presently there is little evidence for a risk of Tdp when used as recommended. Drugs in conditional risk category can cause QTc prolongation and Tdp only under specified conditions of their use. Drugs in special risk (SR) category do not prolong the QT interval per se but have an SR for QTc prolongation because of their other actions. ${ }^{26}$

\section{Statistical Analysis}

Statistical analyzes were performed using SPSS version 21. Comparative analysis was done between two groups of patients (patients with QTc prolongation and patients with normal QTC interval) on days 1,3 , and 5 of ICU admission. Chi-square and $t$ tests were used for categorical and quantitative variables, respectively. Logistic regression models were carried out to calculate the relationship between QTc prolongation and predictors. The $p$ value $\leq 0.05$ was considered statistically significant.

\section{Results}

\section{Baseline Characteristics of Patients}

Two hundred consecutive patients admitted to the MICU, SICU, and EICU were included in this study. Clinical characteristics of the patients are shown in Table 1. The most common cause of admission was diseases of the respiratory system. The mean age of study population was $59.8 \pm 17.9$ years. The mean baseline QTC of patients was $410.5 \pm 36.5 \mathrm{~ms}$.

\section{Incidence of QTc Prolongation on Different Days}

An increasing trend was observed in the incidence of QTC prolongation with increasing length of ICU stay. Incidence of QTC prolongation at ICU admission was 6.5\%. Prolongation of the QTC interval was observed in $9.8 \%$ of patients on day 3 . On day 5 of ICU

Table 1: Characteristics of patients admitted to the ICUs

\begin{tabular}{ll}
\hline N included & 200 \\
Mean age (years) \pm SD & $59.8 \pm 17.9$ \\
Mean weight (kg) & $71.2 \pm 16.0$ \\
Males (\%) & 59 \\
Baseline QTC \pm SD (ms) & $410.5 \pm 36.5$ \\
Mean APACHE II score & $17.9 \pm 5.1$ \\
History of smoking (\%) & $26 \%$ \\
Reason of ICU admission & \\
$\quad$ Diseases of the respiratory system & $38 \%$ \\
$\quad$ Diseases of circulatory system & $19 \%$ \\
$\quad$ Neoplasms & $16.5 \%$ \\
Injury, poisoning, and certain other & $10 \%$ \\
$\quad \begin{array}{l}\text { consequences of external causes } \\
\text { Symptoms, signs, and abnormal clinical and } \\
\text { laboratory findings, not elsewhere classified } \\
\text { Certain infectious and parasitic diseases } \\
\quad \text { Others }\end{array}$ & $4.5 \%$ \\
\hline
\end{tabular}

ICU, intensive care unit; SD, standard deviation; kg, kilogram; APACHE II, acute physiologic and chronic health evaluation 
admission, $15.7 \%$ of patients had prolonged QTc interval. Forty-four patients stayed in the ICUs more than 5 days and the incidence of QTc prolongation was approximately $20 \%$ in these patients.

\section{Comparison of the Two Groups and Assessment of QTc Prolongation Predictors on Day 1 of ICU Admission}

Among 200 patients who were admitted to the ICUs, 13 patients (6.5\%) had prolonged QTc interval and 187 patients (93.5\%) had normal QTc interval (Flowchart 1). All patients with prolonged and normal QTc interval were compared in terms of the risk factors for QTC prolongation. Significant differences were observed between the two groups of patients with respect to low hematocrit (HCT), history of hypertension, $\mathrm{PCl}$, LVEF $<45 \%$, history of mitral regurgitation (MR), alcohol addiction, and ICU admission because of injury, poisoning, and certain other consequences of external causes (Table 2). Logistic regression univariate analysis demonstrated a significant association of QTc prolongation with low HCT, history of PCI, LVEF $<45 \%$, history of MR, ICU admission because of injury, poisoning, and certain other consequences of external causes. From the univariate analysis, those variables with significant $p$ values were included in the logistic regression model for multivariate analysis. History of alcohol addiction and ICU admission due to injury, poisoning, and certain other consequences of external causes were independently associated with QTc prolongation at a statistically significant level (Table 3).

\section{Comparison of the Two Groups and Assessment of QTC Prolongation Predictors on day 3 of ICU Admission}

According to Flowchart 2, QTc prolongation was observed in 17 patients (9.8\%) and 156 patients (90.2\%) had normal QTc interval on day 3 of ICU admission.

Flowchart 1: Patients included in the study on day 1 of intensive care unit admission

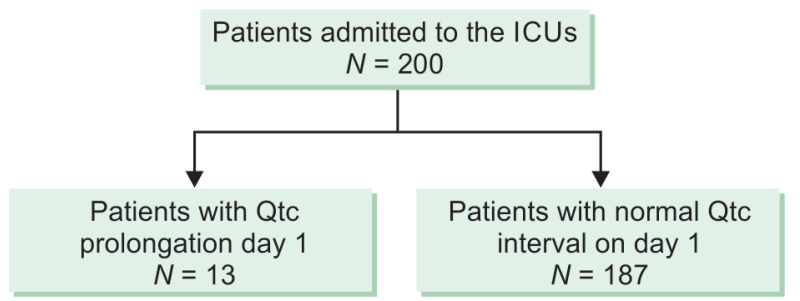

Patients with prolonged and normal QTc interval were compared in terms of risk factors for QTc prolongation and all prescribed medications with QTc-prolongation effect. Azithromycin administration was significantly higher in patients with QTC prolongation compared to other patients (Table 4). It was not significantly different between the two groups of patients in terms of other risk factors and administered drugs. Univariate analysis demonstrated a significant association between administration of azithromycin and QTC prolongation on day 3 of ICU admission (Table 5)

\section{Comparison of the Two Groups and Assessment of QTc Prolongation Predictors on Day 5 of ICU Admission}

Among 121 patients, QTc prolongation were observed in 19 patients (15.7\%; Flowchart 3). Creatinine level above $1.3 \mathrm{mg} / \mathrm{dL}$ and hospitalization in EICU was significantly different between patients with QTc prolongation compared to other patients 5 days after ICU hospitalization (Table 6). High serum creatinine and hospitalization in EICU had statistically significant association with QTc prolongation. Both risk factors were entered into multivariate analysis. They were independently associated with QTc prolongation on day 5 at a statistically significant level (Table 7).

Table 2: Comparison of patients with QTc prolongation and patients with normal QT interval on day 1

\begin{tabular}{llcc}
\hline & $\begin{array}{l}\text { QTc interval } \\
\text { prolongation (\%) }\end{array}$ & $\begin{array}{l}\text { Normal QTc } \\
\text { interval (\%) }\end{array}$ & p value \\
\hline Low HCT (<36\%) & 76.9 & 42.3 & 0.02 \\
Hypertension & 61.5 & 33.7 & 0.04 \\
PCI & 23.1 & 5.3 & 0.01 \\
LVEF $<45 \%$ & 30.8 & 10.7 & 0.03 \\
PVC & 7.7 & 0.5 & 0.01 \\
MR & 15.4 & 1.1 & $<0.001$ \\
Alcohol abuser & 7.7 & 0.5 & 0.01 \\
Injury, poisoning, and & 38.5 & 8 & $<0.001$ \\
certain other consequences & & & \\
of external causes & & & \\
\hline
\end{tabular}

$\mathrm{HCT}$, hematocrit; $\mathrm{PCl}$, percutaneous coronary intervention; LVEF, left ventricular ejection fraction; PVCs, premature ventricular contractions; MR, mitral regurgitation.

Table 3: Logistic regression for predictors of QTc prolongation on the first day of ICU admission

\begin{tabular}{|c|c|c|c|c|c|c|}
\hline \multirow[b]{2}{*}{ Patient characteristics } & \multicolumn{3}{|c|}{ Univariate analysis } & \multicolumn{3}{|c|}{ Multivariate analysis } \\
\hline & $p$ value & OR & $\mathrm{Cl}$ & $p$ value & OR & $\mathrm{Cl}$ \\
\hline Low HCT & 0.03 & 4.56 & $(1.21 \pm 17.10)$ & 0.06 & 4.99 & $(0.93 \pm 26.68)$ \\
\hline Hypertension & 0.05 & 3.15 & $(0.99 \pm 10.02)$ & 0.12 & 0.33 & $(0.08 \pm 1.32)$ \\
\hline $\mathrm{PCl}$ & 0.02 & 5.31 & $(1.26 \pm 22.39)$ & 0.17 & 0.23 & $(0.03 \pm 1.87)$ \\
\hline LVEF $<45 \%$ & 0.04 & 3.71 & $(1.05 \pm 13.16)$ & 0.25 & 0.35 & $(0.06 \pm 2.07)$ \\
\hline PVC & 0.06 & 15.50 & $(0.91 \pm 263.34)$ & 0.09 & 0.07 & $(0.003 \pm 1.46)$ \\
\hline MR & 0.01 & 16.81 & $(2.16 \pm 130.93)$ & 0.09 & 9.93 & $(0.68 \pm 145.73)$ \\
\hline Alcohol abuser & 0.05 & 15.50 & $(0.91 \pm 263.34)$ & 0.01 & 71.30 & $(2.85 \pm 1784.32)$ \\
\hline $\begin{array}{l}\text { Injury, poisoning, and certain other } \\
\text { consequences of external cause }\end{array}$ & 0.002 & 7.17 & $(2.08 \pm 24.66)$ & 0.004 & 10.13 & $(2.05 \pm 49.95)$ \\
\hline
\end{tabular}

$\mathrm{HCT}$, hematocrit; $\mathrm{PCl}$, percutaneous coronary intervention; LVEF, left ventricular ejection fraction; $\mathrm{PVCs}$, premature ventricular contractions; $\mathrm{MR}$, mitral regurgitation; $\mathrm{OR}$, odds ratio; $\mathrm{Cl}$, confidence interval 
Flowchart 2: Patients included in the study on day 3 of intensive care unit admission

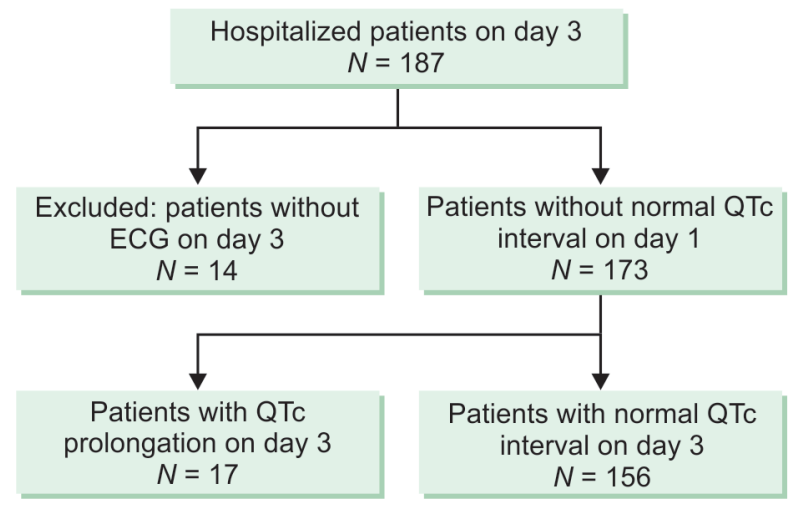

Table 4: Comparison of patients with QTC prolongation and patients with normal QT interval on day 3

\begin{tabular}{llll}
\hline & $\begin{array}{l}\text { QTcinterval } \\
\text { prolongation } \\
(n=17 / 173=\end{array}$ & $\begin{array}{l}\text { No QTcinterval } \\
\text { prolongation } \\
(n=156 / 173=\end{array}$ & \\
Patient & $90.2 \%)$ & p value \\
characteristics & $9.8 \%)$ & $2 \%$ & 0.001 \\
\hline Azithromycin & $17.6 \%$ & & \\
\hline
\end{tabular}

Table 5: Logistic regression for drugs and predictors of QTc prolongation on day 3 of intensive care unit admission

\begin{tabular}{llll}
\hline Variable & $p$ value & $O R$ & $C l$ \\
\hline Azithromycin & 0.006 & 10.93 & $(2.01 \pm 59.30)$ \\
\hline
\end{tabular}

\section{Adverse Outcome Associated with QTc Prolongation}

Patients with QTc prolongation were followed to determine clinical adverse outcomes. Six patients expired in QTc prolonged group. Among these, three patients died because of cardiac arrest without ventricular tachycardia. Two patients died after ventricular tachycardia and ventricular fibrillation. Another patient expired due to multiple organ failure. The resuscitation of these patients was not successful. Mortality rates were compared between the two groups (normal QTc vs prolonged QTc). Mortality rate was higher in patients with QTc prolongation, but this difference was not significant (Table 8).

\section{Discussion}

Current study showed that the incidence of QTc prolongation increased from 6.5 to $15.7 \%$ in ICUs patients during first 5 days of admission. The QTc prolongation was significantly associated with low HCT, history of PCl, LVEF $<45 \%$, history of MR, ICU admission because of injury, poisoning, and certain other consequences of external causes on day 1 of ICU admission and before prescription of any drug in ICU. The APACHE II score, bradycardia, female gender, electrolyte abnormality, DVT, and other comorbidities were not associated with QTc prolongation at this point.

According to the current findings, QTc prolongation is associated with $\mathrm{HCT}<35 \%$. Recent studies revealed anemia can increase the risk of QTc prolongation. ${ }^{27}$ Low HCT can indicate insufficient supply of healthy red blood cells (anemia), large number of white blood cells because of long-term infection, lymphoma, or leukemia. Since low HCT is not the only predictor of anemia, ${ }^{28}$
Flowchart 3: Patients included in the study on day 5 of intensive care unit admission

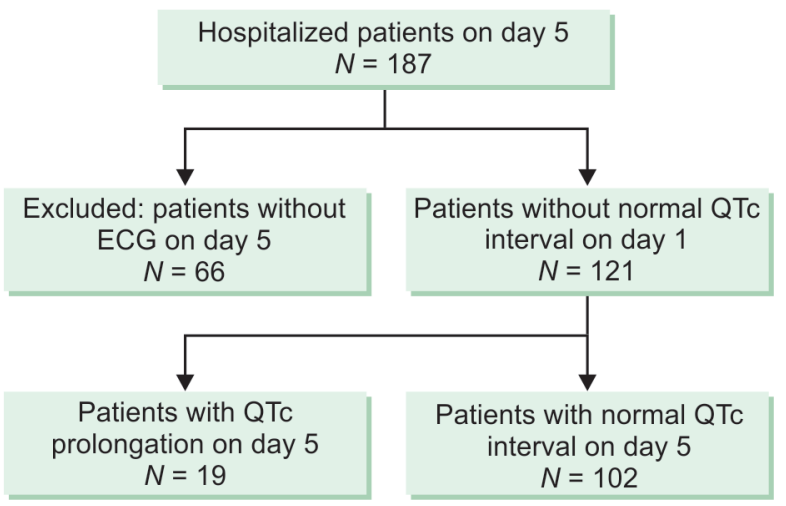

Table 6: Comparison of patients with QTc prolongation and patients with normal QT interval on day 5

\begin{tabular}{llll}
\hline & $\begin{array}{l}\text { No QTc interval } \\
\text { QTeinterval prolon- }\end{array}$ & $\begin{array}{l}\text { prolongation } \\
(n=102 / 121=\end{array}$ & \\
$\begin{array}{l}\text { Patient } \\
\text { characteristics }\end{array}$ & $=15.7 \%)$ & $84.3 \%)$ & $p$ value \\
\hline $\begin{array}{l}\text { High creatinine } \\
(>1.3 \mathrm{mg} / \mathrm{dL})\end{array}$ & $63.2 \%$ & $34.3 \%$ & 0.02 \\
EICU & $57.9 \%$ & $28.4 \%$ & 0.01 \\
\hline
\end{tabular}

EICU, emergency intensive care unit

our results cannot directly demonstrate the association of QTC prolongation with anemia. More studies are required to establish the importance of low HCT as a risk factor for QTc prolongation.

History of hypertension has been implicated as a risk factor for prolonged QTc interval. Some evidences indicate that hypertension may cause left ventricular hypertrophy that can increase the risk of QTc prolongation. ${ }^{29}$ In the current study, history of hypertension was significantly more frequent in patients with QTc prolongation; however, the association between QTc prolongation and history of hypertension was slightly below the level of significance ( $p$ value $=0.05$; Table 3 ).

Few published data exist regarding the association between QTc prolongation and $\mathrm{PCl}$. Some studies reported that post-PCI QTc interval was significantly longer than pre-PCl. ${ }^{30}$ History of $\mathrm{PCl}$ in the current study was associated with QTc prolongation. This finding shows that $\mathrm{PCl}$ may be a predictor of QTc prolongation. Our results also support that LVEF $<45 \%$ can increase the risk of QTc prolongation in ICUs. Therefore, another issue that should be considered in ICUs is the association of LVEF $<45 \%$ with QTC prolongation. Although there are few studies about the association between MR and QTc prolongation, current observation supports the fact that MR can be one of the risk factors for QTc prolongation. Chronic ischemic MR is related to increased risk of heart failure. ${ }^{31}$ It may be hypothesized that heart failure caused by chronic ischemic MR may lead to prolonged QTc interval.

The ICU admission because of injury, poisoning, and certain other consequences of external causes were associated with QTc prolongation. In the current study, patients hospitalized for coronary artery bypass grafting (80\%), tracheal rapture $(10 \%)$, and warfarin toxicity $(5 \%)$ were classified in this category of diseases according to ICD-10. ${ }^{25}$ There are few published studies about the association between QTc prolongation and reason for ICU 
Incidence and Predictors of QTc Prolongation in ICUs

Table 7: Logistic regression for drugs and predictors of QTc prolongation on day 5 of intensive care unit admission

\begin{tabular}{|c|c|c|c|c|c|c|}
\hline \multirow[b]{2}{*}{ Variable } & \multicolumn{3}{|c|}{ Univariate analysis } & \multicolumn{3}{|c|}{ Multivariate analysis } \\
\hline & $p$ value & OR & $\mathrm{Cl}$ & $p$ value & OR & $\mathrm{Cl}$ \\
\hline EICU & 0.02 & 3.34 & $(1.26 \pm 9.45)$ & 0.02 & 3.44 & $(1.23 \pm 9.68)$ \\
\hline $\begin{array}{l}\text { Creatinine high } \\
\text { (>1.3 mg/dL) }\end{array}$ & 0.02 & 3.28 & $(1.19 \pm 9.08)$ & 0.03 & 3.27 & $(1.15 \pm 9.29)$ \\
\hline
\end{tabular}

EICU, emergency intensive care unit

Table 8: Comparison mortality of patients with QTc prolongation and patients with normal QT-interval during intensive care unit admission

\begin{tabular}{llll}
\hline $\begin{array}{l}\text { Patient } \\
\text { characteristics }\end{array}$ & $\begin{array}{l}\text { QTc interval } \\
\text { prolongation }\end{array}$ & $\begin{array}{l}\text { No QTc interval } \\
\text { prolongation }\end{array}$ & p value \\
\hline Mortality & $45.5 \%$ & $33.8 \%$ & 0.28 \\
\hline
\end{tabular}

admission. Our finding shows that the reason of ICU admission can be a predictor of QTc prolongation. According to the above findings, the patients with risk factors that may lead to QTc prolongation on day 1 of ICU admission should be evaluated in terms of QTc interval particularly before prescribing any of QTc-prolonging drugs.

Several classes of medications that prolong the QTc interval including fluoroquinolones and macrolides were prescribed in the ICUs. The Tdp, prolongation of the QTc interval, and sudden death are well defined as undesirable effects of all macrolides. ${ }^{32}$ In addition, different case reports were published about arrhythmias induced by azithromycin from 2001 to 2007. ${ }^{33}$ In 2013, the US Food and Drug Administration also released a warning regarding the prescription of this medication. ${ }^{34}$ However, there is no enough information about this issue. In 2014, one study published about the association between the use of azithromycin and the risk of cardiac arrhythmia and death. In this study, follow-up times were separated into the first 5 days and days 6 through 10 after prescribing azithromycin. They found that azithromycin increased the risks of mortality and arrhythmia on days 1 to 5; however, the association between azithromycin and these adverse effects was not significant from day 6 to $10 .^{35}$ The algorithm for prevention of QTc prolongation recommends evaluation of baseline 12 lead ECG for the patients with more than two risk factors of QTc prolongation and receiving azithromycin. If the QTc interval is prolonged, QTc should be monitored daily for at least 72 hours. ${ }^{36}$ Current results showed the association between QTc prolongation and administration of azithromycin on day 3 of ICU admission. According to this result, we recommend the assessment of benefits and risks of azithromycin therapy for each patient and QTc monitoring for at least 3 days of ICU admission.

One study demonstrated that serum creatinine is higher in patients with QTc prolongation compared with patients without QTc prolongation. ${ }^{37}$ Present results also demonstrated that high serum creatinine may increase the risk of QTc prolongation on 5 days of ICU admission. Although high serum creatinine level does not always represent the kidney disease, it may indicate a problem in elimination of drugs, resulting in increased level of unwanted drug concentrations and hence side effects and toxicities. ${ }^{38}$

There is scarce information about the QTc interval prolongation in patients admitted to EICU. ${ }^{39}$ Most of the research are conducted in MICU, SICU, or CCU. There is a hypothesis that the risk of QTC prolongation is higher in EICU because acutely ill patients with various comorbidities are admitted to the emergency department. In addition, patients in this department may present with the conditions such as drug overdose or other problems that may increase the risk of QTc prolongation. ${ }^{40}$ In the present study, QTC prolongation was associated with EICU admission. Physicians in EICU should be aware of medications with QTc-prolongation effect. They should also monitor patients with a history that may contribute to QTc prolongation.

Our study has some limitations. First, we only evaluated the drugs that were prescribed during ICU stay. In some cases reaching to the complete list of patient's medications before admission to the ICU is not practical. Therefore, we could not analyze the relationship between the medications received before ICU admission and QTC prolongation on day 1 of ICU admission. Another limitation of this study was that nearly one third of our patients did not have ECG on the fifth day (Flowchart 3). A group of patients who were either discharged from ICUs or transferred to the ward were not followed and excluded (42 patients). Some patients died between day 3 and 5 ( 3 patients), while in some patients the ECG was not done since there was no indication to do the ECG (21 patients). Being an observational study, we could not get ECGs done for these patients. The third limitation was that we followed the patients who stayed in the ICU for more than 5 days. From 5 th to 30th day of hospitalization, 44 patients remained in the ICUs. The incidence of QTc prolongation in these patients was evaluated; however, the predictors of QTc prolongation were not identified due to the low number of patients. We recommend conducting a study with a higher number of patients in the future.

\section{Conclusion}

Incidence and predictors of QTc prolongation can be different based on the length of ICU stay. High-risk patients admitted to ICU should be monitored with ECG to reduce the risk of mortality due to life-threatening arrhythmia. Both patient's risk factors and medications that may lead to QTC prolongation in ICU should be assessed separately for the patients with different lengths of hospitalization stay.

\section{References}

1. World Health Organization, News room. Available from: https://www. who.int/en/news-room/fact-sheets/detail/cardiovascular-diseases(cvds)/. Accessed May 17, 2017.

2. Reinelt P, Karth G, Geppert A, Heinz G. Incidence and type of cardiac arrhythmias in critically ill patients: a single center experience in a medical-cardiological ICU. Intensive Care Med 2001;27(9):1466-1473. DOI: $10.1007 / \mathrm{s} 001340101043$.

3. Armahizer MJ, Seybert AL, Smithburger PL, Kane-Gill SL. Drug-drug interactions contributing to QT prolongation in cardiac intensive care units. J Crit Care 2013;28(3):243-249. DOI: 10.1016/j.jcrc.2012. 10.014 .

4. Crouch MA, Limon L, Cassano AT. Clinical relevance and management of drug-related QT interval prolongation. Pharmacotherapy 2003;23(7):881-908. DOI: 10.1592/phco.23.7.881.32730. 
5. Nelson S, Leung J. QTc prolongation in the intensive care unit. AACN Adv Crit Care 2011;22(4):289-295. DOI: 10.4037/ $\mathrm{NCl} .0 \mathrm{~b} 013 \mathrm{e} 31822 \mathrm{db} 49 \mathrm{~d}$.

6. Kallergis EM, Goudis CA, Simantirakis EN, Kochiadakis GE, Vardas PE. Mechanisms, risk factors, and management of acquired long QT syndrome: a comprehensive review. Sci World J. 2012;2012:1-8. DOI: 10.1100/2012/212178.

7. $\mathrm{Ng} \mathrm{TMH}$, Olsen KM, McCartan MA, Puumala SE, Speidel KM, Miller $M A$, et al. Drug-induced QTc-interval prolongation in the intensive care unit: incidence and predictors. J Pharm Pract 2010;23(1):19-24. DOI: $10.1177 / 0897190009356549$.

8. Lam YWF. Drugs and QT interval prolongation in patients with PTSD. The Brown University Psychopharmacol 2019;30(2):2-3. DOI: 10.1002/ pu.30391.

9. Vincenzi FF. Fatal drowning associated with QT prolongation caused by alcohol, some drugs, and congenital long QT syndrome: the role of the diving reflex. EC. Pharmacol Toxicol 2018;6(7):622-633.

10. Gowda RM, Khan IA, Wilbur SL, Vasavada BC, Sacchi TJ. Torsade de pointes: the clinical considerations. Int J Cardio 2004;96(1):1-6. DOI: 10.1016/j.ijcard.2003.04.055.

11. Zhang Y, Ouyang P, Post WS, Dalal D, Vaidya D, Blasco-Colmenares $\mathrm{E}$, et al. Sex-steroid hormones and electrocardiographic QT-interval duration: findings from the third national health and nutrition examination survey and the multi-ethnic study of atherosclerosis. Am J Epidemiol 2011;174(4):403-411. DOI: 10.1093/aje/kwr172.

12. Carnethon MR, Anthony MS, Cascio WE, Folsom AR, Rautaharju PM, Liao $D$, et al. A prospective evaluation of the risk of QT prolongation with hormone replacement therapy: the atherosclerosis risk in communities study. Ann Epidemiol 2003;13(7):530-536. DOI: 10.1016/ s1047-2797(03)00050-4.

13. Charbit B, Christin-Maître S, Démolis J-L, Soustre E, Young J, FunckBrentano $C$. Effects of testosterone on ventricular repolarization in hypogonadic men. Am J Cardiol 2009;103(6):887-890. DOI: 10.1016/j. amjcard.2008.11.041.

14. Ma T, Cai J, Zhu Y-S, Chu X-F, Wang Y, Shi G-P, et al. Association between a frailty index based on common laboratory tests and QTC prolongation in older adults: the rugao longevity and ageing study. Clin Interv Aging 2018;13:797-804. DOI: 10.2147/CIA.S149791.

15. Pickham D, Flowers E, Drew BJ. Hyperglycemia is associated with corrected QT prolongation and mortality in acutely ill patients. J Cardio Nurs 2014;29(3):264. DOI: 10.1097/JCN.0b013e31827f174c.

16. Hegyi B, Borst JM, Lucena AJ, Bailey LRJ, Bossuyt J, Bers DM. Diabetic hyperglycemia regulates potassium channels and arrhythmias in the heart via autonomous CaMKII activation by O-linked glycosylation. Biophys J 2019;116(3):98a. DOI: 10.1016/j.bpj.2018.11.566.

17. Fernandes FM, Silva EP, Martins RR, Oliveira AG. QTc interval prolongation in critically ill patients: prevalence, risk factors and associated medications. PLoS ONE 2018;13(6):e0199028. DOI: 10.1371/ journal.pone.0199028.

18. Beitland S, Platou ES, Sunde K. Drug-induced long QT syndrome and fatal arrhythmias in the intensive care unit. Acta Anaesthesiol Scandinavica 2014;58(3):266-272. DOI: 10.1111/aas.12257.

19. Tisdale JE, Wroblewski HA, Overholser BR, Kingery JR, Trujillo TN, Kovacs RJ. Prevalence of QT interval prolongation in patients admitted to cardiac care units and frequency of subsequent administration of QT interval-prolonging drugs. Drug Safe 2012;35(6):459-470. DOI: 10.2165/11598160-000000000-00000.

20. Smithburger PL, Seybert AL, Armahizer MJ, Kane-Gill SL. QT prolongation in the intensive care unit: commonly used medications and the impact of drug-drug interactions. Expert Opin Drug Safe 2010;9(5):699-712. DOI: 10.1517/14740331003739188.

21. Zarowitz BJ, Tisdale JE. Navigating the minefield of QTc intervalprolonging therapy in nursing facility residents. J Am Geriatr Soci 2019;67(7):1-8. DOI: 10.1111/jgs.15810.

22. Indik JH, Pearson EC, Fried K, Woosley RL. Bazett and fridericia QT correction formulas interfere with measurement of drug-induced changes in QT interval. Heart Rhythm 2006;3(9):1003-1007. DOI: 10.1016/j.hrthm.2006.05.023.

23. Luo S, Michler K, Johnston P, Macfarlane PW. A comparison of commonly used QT correction formulae: the effect of heart rate on the QTc of normal ECGs. J Electrocardiol 2004;37(Suppl. 1):81-90. DOI: 10.1016/j.jelectrocard.2004.08.030.

24. Manini AF, Stimmel B, Vlahov D. Racial susceptibility for QT prolongation in acute drug overdoses. J Electrocardiol 2014;47(2): 244-250. DOI: 10.1016/j.jelectrocard.2013.12.002.

25. International Statistical Classification of Diseases and Related Health Problems 10th Revisione. WHO. Available from: https://icd.who.int/ browse10/2016/en/. Accessed April 17, 2018.

26. Incredible Medicine. Available from: https://crediblemeds.org/. Accessed March 18, 2018.

27. Khode VH, Kammar KF. QTc changes in non-pregnant females with severe iron deficiency anaemia. J Clin Diagnostic Res 2012;6(5): 777-779.

28. Hematocrit test. Mayoclinic. Available from: https://www.mayoclinic. org/tests-procedures/hematocrit/about/pac-20384728. Accessed February 8, 2019.

29. Klimas J, Stankovicova T, Kyselovic J, Bacharova L. Prolonged QT interval is associated with blood pressure rather than left ventricular mass in spontaneously hypertensive rats. Clin Exp Hypertens 2008;30(7):475-485. DOI: 10.1080/10641960802443399.

30. Haarmark C, Hansen PR, Vedel-Larsen E, Haahr Pedersen S, Graff C, Andersen MP, et al. The prognostic value of the Tpeak-Tend interval in patients undergoing primary percutaneous coronary intervention for ST-segment elevation myocardial infarction. J Electrocardiol 2009;42(6):555-560. DOI: 10.1016/j.jelectrocard.2009.06.009.

31. Pierard LA, Carabello BA. Ischaemic mitral regurgitation: pathophysiology, outcomes and the conundrum of treatment. Eur Heart J 2010;31(24):2996-3005. DOI: 10.1093/eurheartj/ehq411.

32. Russo V, Puzio G, Siniscalchi N. Azithromycin-induced QT prolongation in elderly patient. Acta Bio Medica Atenei Parmensis 2006;77(1):30-32.

33. Huang B-H, Wu C-H, Hsia C-P, Yin Chen C. Azithromycin-induced torsade de pointes. Pacing Clin Electrophysiol 2007;30(12):1579-1582. DOI: 10.1111/j.1540-8159.2007.00912.x.

34. Administration USF and D. FDA drug safety communication: Azithromycin (Zithromax or Zmax) and the risk of potentially fatal heart rhythms. Drug Safety Communications. Available from: https://www.fda.gov/drugs/drug-safety-and-availability/fda-drugsafety-communication-azithromycin-zithromax-or-zmax-and-riskpotentially-fatal-heart. Accessed March 18, 2019.

35. Rao GA, Mann JR, Shoaibi A, Bennett CL, Nahhas G, Sutton SS, et al. Azithromycin and levofloxacin use and increased risk of cardiac arrhythmia and death. Ann Fam Med 2014;12(2):121-127. DOI: 10.1370/ afm.1601.

36. Guidelines TOSUWMC clinical practice. QT prolongation guidelines. Available from: https://evidencebasedpractice.osumc.edu/ Documents/Guidelines/QTc \%0AProlongation and TdP. Pdf. Accessed March 14, 2018.

37. Kim SM, George B, Alcivar-Franco D, Campbell CL, Charnigo R, Delisle $B$, et al. QT prolongation is associated with increased mortality in end stage liver disease. World J Cardiol 2017;9(4):347. DOI: 10.4330/wjc. v9.i4.347.

38. Stevens LA, Schmid CH, Greene T, Li L, Beck GJ, Joffe MM, et al. Factors other than glomerular filtration rate affect serum cystatin $C$ levels. Kidney Int 2009;75(6):652-660. DOI: 10.1038/ki.2008.638.

39. Kumar S, Birda C, Bhalla A, Sharma N, Kumari S. Prevalence and prognostic significance of prolonged QTc interval in emergency medical patients: a prospective observational study. Int J Crit IIIn Inj Sci 2018;8(1):28. DOI: 10.4103/IJCIIS.IJCIIS_59_17.

40. Pourmand A, Mazer-Amirshahi M, Chistov S, Sabha Y, Vukomanovic D, Almulhim M. Emergency department approach to QTC prolongation. am. J Emerg 2017;35(12):1928-1933. DOI: 10.1016/j.ajem.2017. 08.044 . 